J. Austral. Math. Soc. 20 (Series A) (1975), 305-306.

\title{
A NOTE ON PRIME RADICALS OF CERTAIN GROUP RINGS
}

\author{
KENG-TEH TAN
}

(Received 26 April 1974)

Communicated by G. E. Wall

Let $R$ be a ring (with identity) and $P(R)$ denote its prime radical. $R$ is called semi-prime when $P(R)=(0)$. If $G$ is a group, the group ring of $G$ over $R$ will be denoted by $R G$.

In Tan (1974), we ask the following question: if $R$ is left Goldie and $G$ torsionfree abelian, is it true that $P(R G)=P(R) G$ ?

In this note, we will prove that the answer is affirmative. In fact, we will establish the following more general result.

THEOREM. If $R$ is left Goldie and $G$ is torsion-free, then $P(R G)=P(R) G$.

To prove this, we need the following

Lemma 1. If $K$ is an ideal of $R$ such that $R / K$ is semi-prime, then $K \supseteq P(R)$.

Proof. See Lambek (1966), page 56.

Lemma 2 (Connell-Passman). $R G$ is semi-prime if and only if $R$ is semiprime and the order of no finite normal subgroup of $G$ is a zero divisor in $R$.

Proof. See Lambek (1966), Proposition 8, page 162.

We now prove the theorem.

Since $R$ is left Goldie, it follows that $P(R)$ is nilpotent, then so is $P(R) G$. Consequently, $P(R) G \subseteq P(R G)$.

On the other hand, we have

$$
R G / P(R) G \cong(R / P(R)) G=T,
$$

say. However $T$ is semi-prime by Lemma 2 . It then follows by Lemma 1 that

$$
P(R) G \supseteq P(R G) .
$$

This proves the theorem. 


\section{References}

J. Lambek (1968), Lectures on Rings and Modules (Blaisdell, Waltham, 1966).

D. S. Passman (1962), 'Nil ideals in group rings', Mich. Math. J. 9, 375-384.

K. T. Tan (1974), 'Some torsion-free group rings with nilpotent prime radicals', J. Austral. Math. Soc. 18, 372-375.

Department of Mathematics

University of Malaya

Kuala Lumpur, Malaysia. 